\title{
DESIGN, ANALYSIS AND 3D PRINTING OF ARCHITECTED SANDWICH PANELS
}

\author{
H. Yazdani Sarvestani \\ AM3L Laboratory, Department of Bioresource \\ Engineering \\ McGill University \\ Island of Montreal, QC H9X 3 V9 Canada \\ H. Niknam \\ AM3L Laboratory, Department of Bioresource \\ Engineering \\ McGill University \\ Island of Montreal, QC H9X 3 V9 Canada
}

\author{
A.H. Akbarzadeh \\ AM3L Laboratory, Department of Bioresource \\ Engineering \\ McGill University \\ Island of Montreal, QC H9X 3V9 Canada \\ Email: hamid.akbarzaeh@mcgill.ca \\ K. Hermenean \\ MACHINA Corp \\ Edmonton, AB T6H 2 H3 Canada
}

\begin{abstract}
In this study, we implement a finite element approach and conduct experimental impact tests to evaluate the performance of 3D printed lightweight sandwich panels with architected cellular cores of programmable six-sided cells. The standard mechanics homogenization technique is implemented through a finite element modelling to accurately predict the effective mechanical properties of architected cellular cores. We implement an explicit large deformation finite element simulation using ANSYS to analyze the elastoplastic behavior of sandwich panels under a low-velocity impact. To experimentally corroborate the developed computational model and to evaluate the manufacturability of architected sandwich panels, we use the fused deposition modeling to 3D print samples of polylactic acid biopolymers. We conduct low-velocity impact experimental tests on the 3D printed panels to investigate their energy absorption capabilities. The results show that the auxetic sandwich panel is potentially an appropriate candidate for energy absorption applications due to its high energy absorption capability.
\end{abstract}

Keywords-component: Architected 3D printed panels; Cellular solids; Energy absorption; Low-velocity impact.

\section{INTRODUCTION}

Sandwich panels are nowadays extensively used in aerospace, marine, automotive, and windmills industries due to their high flexural stiffness-to-weight ratio, excellent thermal insulation, and high energy absorption capability [1]. Sandwich panels consist of two thin but stiff face-sheets at the top and bottom of the panels separated by a lightweight and relatively thick core. The lightweight core connects the face-sheets with small increase in weight but provides sandwich panels with a high bending stiffness and buckling resistance [2] as well as superb shear stiffness and energy absorption capability [3]. The lightweight core can be made of alternative materials but it is usually in the form of cellular materials, e.g. foams or periodic lattices. In a sandwich panel, the face-sheets carry most of inplane and bending loads while the core mainly carries transverse shear [1].

The energy absorption capability of an architected sandwich panel mainly depends on the material properties and geometrical features of solid sheets and the cellular core. Among all cell topologies for the core of architected sandwich panels, hexagonal honeycomb has been commonly used and analyzed as a cellular core $[4,5]$. Sandwich panels with conventional honeycomb cellular cores are stiff and lightweight, while they absorb high energy under impact and shockwaves for applications in sports goods, automotive, and aerospace $[6,7]$. However, they have some issues due to their closed-cell architectures including gas retention, leading to low thermal conductivity, and moisture trapping [1]. An alternative sandwich core is open-cell cores such as truss-like structures $[8,9]$. It has been shown that truss-like cellular cores, with low relative densities, have significantly higher buckling resistance and energy absorption capability compared to hexagonal honeycomb cellular cores $[10,11]$.

More recently, auxetic sandwich cores have been examined due to their unusual deformation mechanisms, which is negative Poisson's ratio. Auxetic cores possess promising properties in the aforementioned applications compared to other topologies of cellular solids. Due to the auxetic behavior, auxetic cellular cores reveal unique mechanical properties such as increased indentation resistance [12], shear resistance [13], fracture toughness [14, 15], and energy absorption capacity $[16,17]$. Although a few investigations have been performed on architected sandwich panels, e.g. flexural behavior [18-20] and out-of-plane compressive strength [21,22], less attention has been paid to explore the energy absorption capabilities of $3 \mathrm{D}$ printed sandwich panels with architected cellular cores.

The current study focuses on the effect of core's cell topology and relative density on the energy absorption 
capability and structural design of 3D printed sandwich panels with an architected cellular core.

\section{SANDWICH PANEL AND EXPERIMENTAL TEST CONFIGURATION}

Dimensions of the sandwich panel including length $(a)$, width $(b)$, and total thickness $(h)$ are shown in Fig. 1. The coordinate system $(x, y, z)$ is located at the midplane of the sandwich panel. The selected dimensions are listed in Table 1, which are chosen based on ASTM standard D3763 [23] and testing limitations.

TABLE I. GEOMETRIC PARAMETERS OF SANDWICH PANELS (UNIT: MM).

\begin{tabular}{|c|c|c|c|c|}
\hline Length (a) & Width (b) & $\begin{array}{c}\text { Total } \\
\text { thickness (h) }\end{array}$ & $\begin{array}{c}\text { Core } \\
\text { thickness } \\
\text { (tc) }\end{array}$ & $\begin{array}{c}\text { Face-sheet } \\
\text { thickness } \\
\text { (ts) }\end{array}$ \\
\hline 100 & 100 & 50 & 40 & 5 \\
\hline
\end{tabular}

\section{A. Sample preparation}

MK2 3D printer of MACHINA Corp. with the layer height of $0.25 \mathrm{~mm}$ manufactures the architected sandwich panels. MK2 3D printer uses fused deposition modeling technology (FDM) to additively manufacture architected samples of PLA polymers. Two types of sandwich panels (vertical or horizontal cells) with three different topologies of cellular cores (hexagonal, rectangular, and auxetic) are 3D printed.

\section{B. Experimental low-velocity impact test}

The low-velocity impact tests are performed on sandwich panels with different 3D printed cores using a drop weight machine based on the guidelines given in the ASTM standard D3763 [23]. The impactor has a mass of $12 \mathrm{~kg}$ and a diameter of $25.4 \mathrm{~mm}$. During the impact test, the specimens are constrained between two parallel rigid supports with a $75 \mathrm{~mm}$ diameter hole in the center (see Fig. 2).

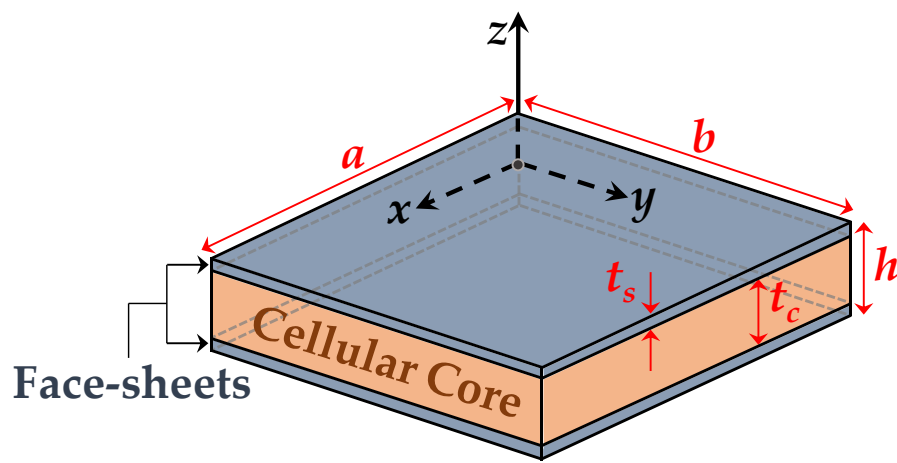

Figure 1. Geometry of an architected sandwich panel and the considered coordinate system.

\section{FINITE ELEMENT MODELING}

The explicit large deformation 3D finite element modeling (FEM) is conducted using the commercial software ANSYS Workbench 18.2. Fig. 3 presents the model developed in ANSYS for conducting the low velocity impact analysis. The face-sheets and the core are meshed with quadrilateral and triangular elements and a convergence study is conducted to avoid mesh size dependency of FEM results. The spherical impactor is defined as a rigid body and the dynamic load is applied by assuming an initial velocity for the rigid impactor. The stress-strain curve of PLA is assumed as an elastoperfectly plastic model for FEM analysis.

\section{RESUlTS AND DISCUSSION}

In this section, we examine the effects of the topology and relative density of cellular core of architected sandwich panels on energy absorption when the sandwich panel is subjected to a low-velocity impact. For experimental study, the sandwich panel is subjected to a $3 \mathrm{~J}$ low-velocity impact test.

\section{A. Energy-time history}

Fig. 4 presents the experimental and numerical results for the energy-time history for alternative cell configuration, cell topology, and cell relative density. There is a good agreement between the results of experimental tests and numerical analyses. In the energy absorption versus time history res, the amounts of absorbed and returned (released) energies during the impact test can be observed. The absorbed energy is the energy mostly dissipated by various failure mechanisms such as delamination and cracking [24]. The returned (released) energy is the elastic energy. Herein, we define the energy performance based on the ratio of absorbed energy / maximum impact energy [24] (see Fig. 4).

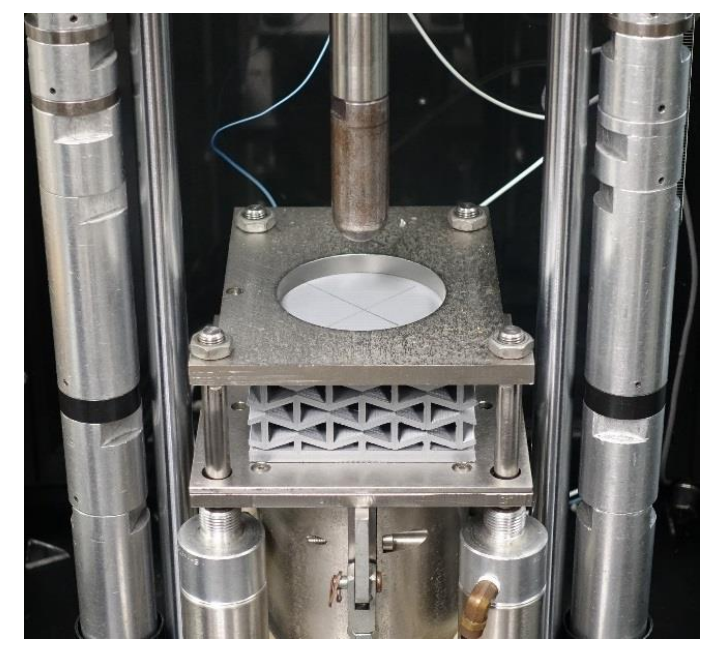

Figure 2. Low-velocity impact test configuration of 3D printed architected sandwich panels. 


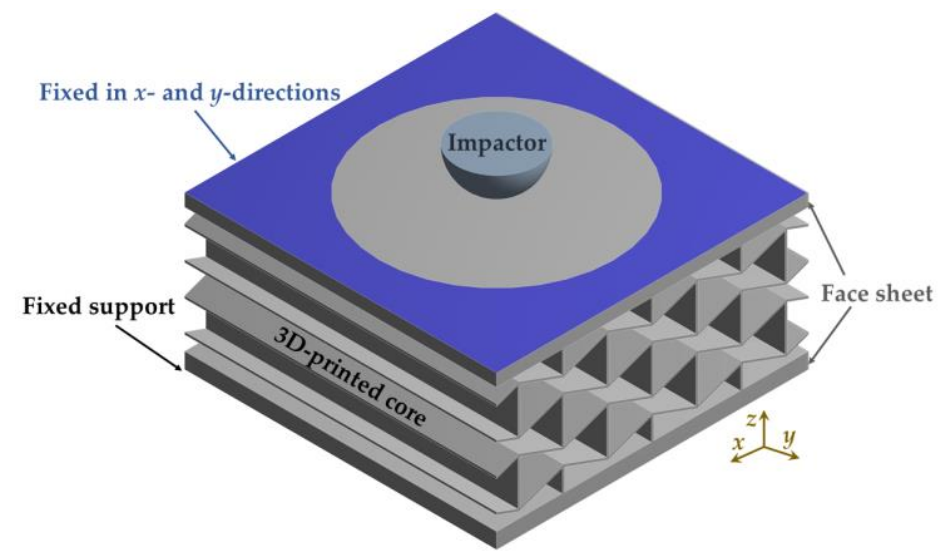

Figure 3. Model developed in ANSYS for the low-velocity impact analysis.
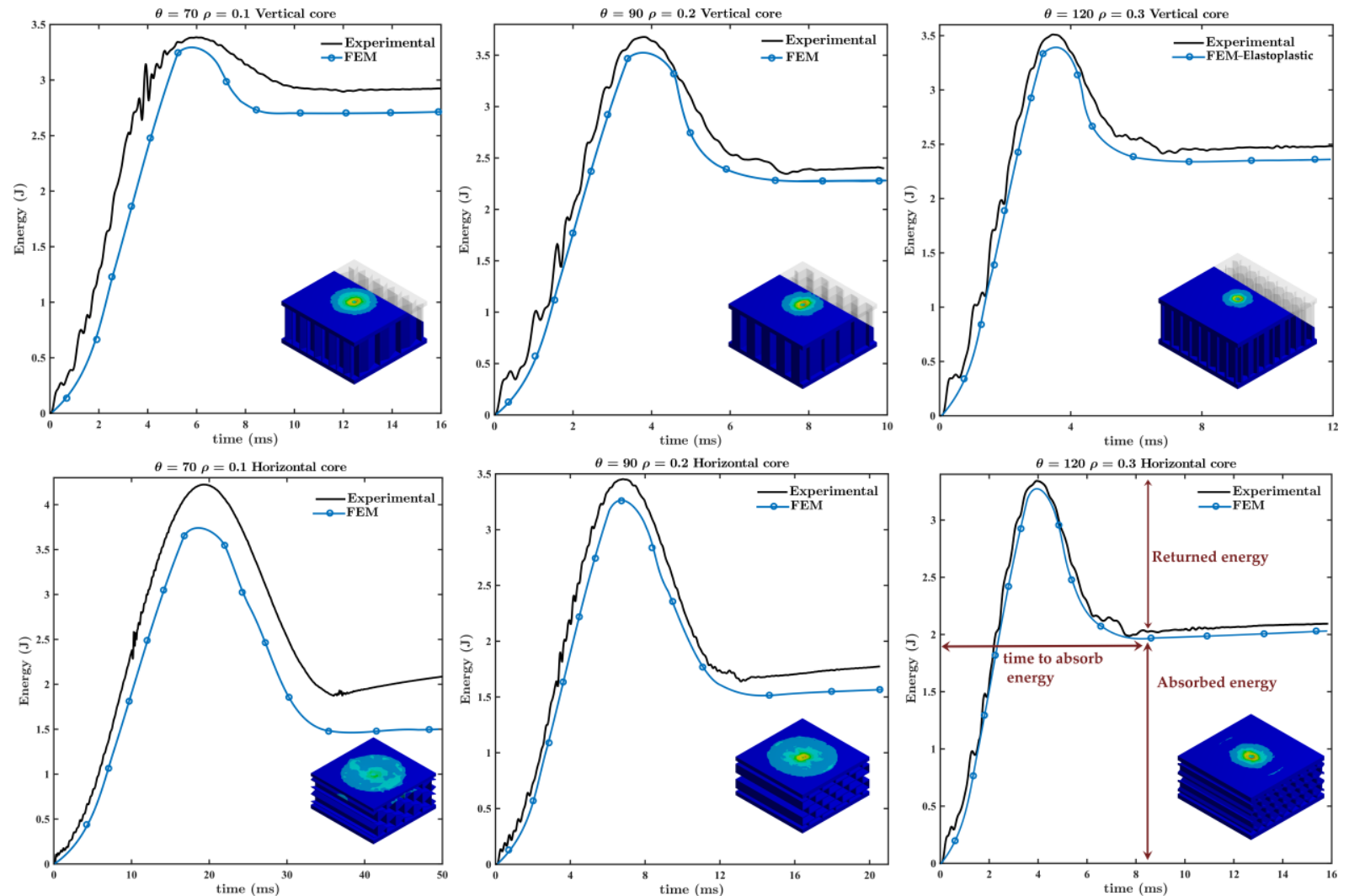

Figure 4. Energy-time history obtained from the experimental impact test on 3D printed architected sandwich panels of alternative cellular core configuration, cell topology, and cell relative density compared with FEM results 

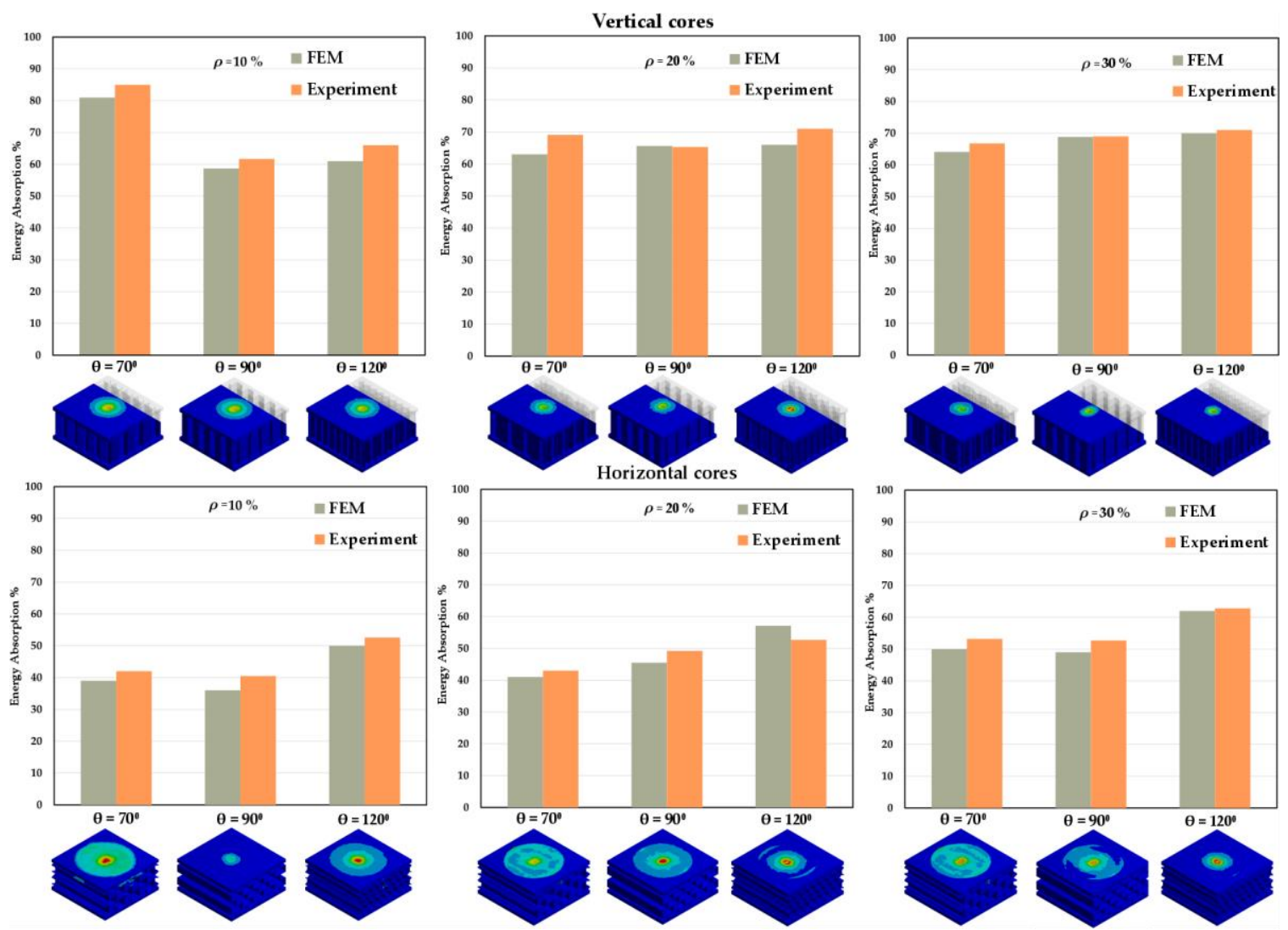

Figure 5. Experimental and FEM results for the energy absorption capability of 3D printed architected sandwich panels of alternative cellular core configuration, cell topology, and cell relative density.

\section{B. Energy absorption capability}

Fig. 5 shows the experimental and numerical energy absorption capability of 18 3D printed sandwich panels with different core topologies including the horizontal and vertical auxetic, rectangular, and hexagonal cores (cell wall angle $\theta=$ $70^{\circ}, 90^{\circ}$, and $120^{\circ}$, respectively); and the relative densities of $\rho$ $=0.1,0.2$, and 0.3. There is a good agreement between the results of experimental tests and numerical analyses. For all considered relative densities of horizontal/vertical cellular cores, the rectangular and hexagonal cellular cores provide a slightly higher energy absorption capability than the auxetic cellular core except for the auxetic cellular core with the relative density of $\rho=0.1$. It should be noted that the auxetic cellular core with the relative density of $\rho=0.1$ in the vertical configuration is the optimum cellular core, in terms of energy absorption, for the architected sandwich panels subjected to specific impact energy. 25\% and 29\% improvements for energy absorption are observed during the experimental tests by using auxetic cellular cores for architected sandwich panels in comparison with hexagonal and rectangular cores, respectively.

\section{CONCLUDING REMARKS}

We develop a numerical and experimental study to determine the optimum geometry of architected 3D printed sandwich panels with a periodic cellular core. We 3D print architected sandwich panels of three different cell topologies made of biopolymers and conduct experimental low-velocity impact tests on samples to estimate their energy absorption capability. The results show that if relative density of the auxetic cellular core is selected appropriately for a specific value of impact energy, the sandwich panels with auxetic cellular cores can have a higher level of energy absorption capability up to $33 \%$ compared to the rectangular and hexagonal cellular sandwich panels.

\section{ACKNOWLEDGMENT}

A.H. Akbarzadeh acknowledges the financial supports provided by McGill University and Natural Science and Engineering Research Council of Canada (NSERC) through NSERC Discovery Grant RGPIN-2016-04716 and NSERC Engage Grant EGP 507009-16.

\section{REFERENCES}

1. Schaedler, T.A. and W.B. Carter, Architected cellular materials. Annual Review of Materials Research, 2016. 46: p. 187-210.

2. Gibson, L.J. and M.F. Ashby, Cellular solids: structure and properties. 1999: Cambridge university press.

3. Allen, H.G., Analysis and design of structural sandwich panels: the commonwealth and international library: structures and solid body mechanics division. 2013: Elsevier.

4. Rathbun, H., et al., Performance of metallic honeycomb-core sandwich beams under shock loading. International journal of solids and structures, 2006. 43(6): p. 1746-1763.

5. Buitrago, B.L., et al., Modelling of composite sandwich structures with honeycomb core subjected to high-velocity impact. Composite structures, 2010. 92(9): p. 2090-2096. 
6. Masters, I. and K. Evans, Models for the elastic deformation of honeycombs. Composite structures, 1996. 35(4): p. 403-422.

7. Becker, W., Closed-form analysis of the thickness effect of regular honeycomb core material. Composite Structures, 2000. 48(1): p. 67-70.

8. $\quad$ Sypeck, D.J., Cellular truss core sandwich structures. Applied Composite Materials, 2005. 12(3): p. 229-246.

9. Hu, Y., et al., Fabrication and mechanical behaviors of corrugated lattice truss composite sandwich panels. Composites Science and Technology, 2016. 125: p. 114-122.

10. Wadley, H.N., N.A. Fleck, and A.G. Evans, Fabrication and structural performance of periodic cellular metal sandwich structures. Composites Science and Technology, 2003. 63(16): p. 2331-2343.

11. Xiong, J., et al., Shear and bending performance of carbon fiber composite sandwich panels with pyramidal truss cores. Acta Materialia, 2012. 60(4): p. 1455-1466.

12. Lakes, R. and K. Elms, Indentability of conventional and negative Poisson's ratio foams. Journal of Composite Materials, 1993. 27(12): p. 1193-1202.

13. Lakes, R., Foam structures with a negative Poisson's ratio. Science, 1987. 235: p. 1038-1041.

14. Yang, W., et al., Review on auxetic materials. Journal of materials science, 2004. 39(10): p. 3269-3279.

15. Grima, J.N. and K.E. Evans, Auxetic behavior from rotating squares. Journal of Materials Science Letters, 2000. 19(17): p. 1563-1565.

16. Alderson, A. and K. Alderson, Auxetic materials. Proceedings of the Institution of Mechanical Engineers, Part G: Journal of Aerospace Engineering, 2007. 221(4): p. 565-575.
17. Hou, S., et al., How does negative Poisson's ratio of foam filler affect crashworthiness? Materials \& Design, 2015. 82: p. 247-259.

18. Altan, G. and V. Kovan, Flexural behavior of $3 D$ printed honeycomb sandwich structures with waste filler material. Materials Testing, 2016. 58(10): p. 833-838.

19. Li, T. and L. Wang, Bending behavior of sandwich composite structures with tunable 3D-printed core materials. Composite Structures, 2017. 175: p. 46-57.

20. Berger, J., H. Wadley, and R. McMeeking, Mechanical metamaterials at the theoretical limit of isotropic elastic stiffness. Nature, 2017. 543(7646): p. 533-537.

21. Dikshit, V., et al., Out of plane compressive strength of $3 D$ printed vertical pillared corrugated core structure. 2016.

22. Turner, A.J., Low-Velocity Impact Behavior of Sandwich Panels with 3D Printed Polymer Core Structures. 2017, Wright State University.

23. Association, U.S., ASTM D3763-2006 Standard Test Method for High Speed Puncture Properties of Plastics using Load and Displacement Sensor. USA Standards Association International, USA, 2006.

24. Ouadday, R., et al., Experimental and numerical investigation on the impact behavior of dual-core composite sandwich panels designed for hydraulic turbine applications. Composite Structures, 2017. 